\title{
Performance of an Energy Detector over Channels with Both Multipath Fading and Shadowing
}

\author{
Saman Atapattu, Student Member, IEEE, Chintha Tellambura, Senior Member, IEEE, \\ and Hai Jiang, Member, IEEE
}

\begin{abstract}
This paper analyzes the performance of an energy detector over wireless channels with composite multipath fading and shadowing effects. These effects are modeled by using the $K$ and $K_{G}$ channel models. Closed-form average detection probabilities are derived for both $K$ and $K_{G}$ channel models for the no-diversity reception case. A simple approximation is also derived for large values of energy threshold in the energy detector. The analysis is then extended to cases with diversity receptions including maximal ratio combining (MRC) and selection combining (SC). Analytical results are verified by Monte Carlo simulation and by numerical methods. Receiver operating characteristic (ROC) curves are presented for different degrees of multipath fading and shadowing. Finally, the Rayleigh-lognormal distribution and the $K$ distribution are numerically compared, and the validity of the $K$ channel model for representing the impact of shadowing on the performance of energy detection is affirmed.
\end{abstract}

Index Terms-Energy detector, detection probability, fading, shadowing.

\section{INTRODUCTION}

$\mathbf{S}$ IGNAL detection is essential for wireless communications. Typical applications include radar detection, carriersense multiple access (CSMA) based networks, and cognitive radio (to detect the presence of licensed users). Traditional signal detection techniques involve the energy detector, the matched filter, or the feature detector. Among them, the energy detector has the lowest hardware complexity. As a non-coherent device, an energy detector samples the received signal energy over a time window, compares with a prespecified threshold, and determines the presence or absence of an unknown signal [1]. Energy detector does not require any prior knowledge of the transmitted signal and fading channel. Therefore, it can be found in practical communication systems such as radar detection and orthogonal frequency division multiplexing (OFDM) applications. Low-complexity and non-coherent energy detection has thus been applied in ultra-wideband (UWB) wireless communication networks [2].

In the open literature, many research efforts have been expended to analyze the performance of energy detectors. In [3], the statistics of the presence and absence of an

Manuscript received July 14, 2009; revised January 13, 2010; accepted September 14,2010 . The associate editor coordinating the review of this paper and approving it for publication was M. Torlak.

This work was supported by the Natural Science and Engineering Research Council (NSERC) of Canada under a strategic grant, and the Alberta Innovates-Technology Futures, Alberta, Canada under a New Faculty Award.

The authors are with the Department of Electrical and Computer Engineering, University of Alberta, Edmonton, AB, Canada T6G 2V4 (e-mail: \{atapattu, chintha, hai.jiang\}@ece.ualberta.ca).

Digital Object Identifier 10.1109/TWC.2010.100110.091042 unknown deterministic signal are formulated as non-central and central chi-square distributions, respectively, assuming a flat, band-limited, Gaussian noise channel. The detection probability $\left(P_{d}\right)$ and false alarm probability $\left(P_{f}\right)$ are also derived. Based on the detection and false alarm probabilities, analytical results are given in [4] for Rayleigh, Rice and Nakagami fading channels. A different analytical approach is given in [5] [6] for the performance of an energy detector with no diversity for Rayleigh, Rice and Nakagami fading channels and with different diversity receptions such as maximal ratio combining (MRC), selection combining (SC) and switch-andstay combining (SSC). Recently, the performance with equal gain combining (EGC) under a Nakagami fading channel is analyzed in [7]. In our work [8], an energy detector is investigated in relay-based cognitive radio networks. Further, we have investigated the performance of an energy detector over $\eta$ - $\mu$ fading channel in [9]. All the above research efforts have focused on multipath fading only.

Apart from the multipath fading, wireless signals also undergo shadowing. While the multipath fading can be modeled as a Rayleigh, Rice or Nakagami distribution, shadowing process is typically modeled as a lognormal distribution [10]. Therefore, some practical communication channels can be modeled as multipath fading superimposed on lognormal shadowing. Due to the difficulty of analyzing digital wireless communication systems over composite fading models, the shadowing effect is sometimes neglected in the literature.

Recently, to model channels with composite multipath fading and shadowing, the $K$ distribution and generalized $K$ (termed $K_{G}$ ) distribution have been introduced, by using a gamma distribution to approximate the lognormal distribution of the shadowing. In [11], the $K$ distribution, a mixture of Rayleigh distribution and gamma distribution, is used to approximate the Rayleigh-lognormal distribution in a real channel, referred to as the $K$ channel model. The accuracy of the approximation is verified by comparison of their moment generating functions (MGFs). In [12], the $K_{G}$ distribution, a mixture of the Nakagami distribution and gamma distribution, is presented to approximate the Nakagami-lognormal distribution in a real channel, referred to as the $K_{G}$ channel model. The $K$ and $K_{G}$ channel models have been well adopted for analysis of wireless communication systems with both multipath fading and shadowing. The $K_{G}$ model includes special cases, such as the $K$ model, and can also approximate the Nakagami- $m$ model, the Rayleigh-lognormal (RL) distribution and the Suzuki model [11]. This model may also be used for tropospheric propagation of radio, radar clutter and 
optical scintillation. The bit error rate (BER) performance is derived in [13] for differential phase-shift keying (DPSK) and minimum shift keying (MSK), using the $K$ channel model. In [14], the outage probability with and without co-channel interference is presented, based on the $K_{G}$ channel model, and the results match well with the Nakagami-lognormal model. In [15], signal-to-noise ratio (SNR) statistics, the average Shannon's channel capacity and the BER performance are studied, based on the $K_{G}$ channel model. Average BER with different diversity receptions can be found in [16], with the $K_{G}$ channel model. Recently, performance of generalized selection combining receivers over the $K$ channel model is presented in [17], based on marginal MGF of the end-to-end SNRs. However, the performance of an energy detector in $K_{G}$ channels is not available in the literature.

In this research, we analyze the performance of an energy detector for channels with both multipath fading and shadowing, by adoption of the $K$ and $K_{G}$ channel models. The critical part of the analysis is the derivation of the average detection probability. This derivation requires the generalized MarcumQ function be averaged over the $K_{G}$ distribution. Since the $K$ and $K_{G}$ channel models contain modified Bessel functions, direct integration appears intractable or does not seem to lead to simple closed-form solutions. In order to circumvent these difficulties, we use the following 'trick.' The $K$ or $K_{G}$ channel model actually is a result of averaging a conditional Rayleigh or Nakagami probability density function (PDF) by a gamma PDF. Therefore, we use existing results on the energy detector for Nakagami- $m$ fading case and average them over the gamma PDF (which models the shadowing part). This simple trick allows us to avoid the averaging over the modified Bessel function of the third kind. Similar approaches can be applied for the diversity combining techniques with each diversity branch having identical instantaneous shadowing effect.

The rest of the paper is organized as follows. The system model is described in Section II. Average detection probability of an energy detector is analyzed in Sections III and IV for cases with no-diversity reception and diversity reception, respectively. Numerical and simulation results are presented in Section V. The concluding remarks are made in Section VI.

\section{SySTEM Model}

The received signal at an energy detector at time $t$ can be represented as:

$$
r(t)= \begin{cases}w(t) ; & H_{0} \\ h s(t)+w(t) ; & H_{1}\end{cases}
$$

where $h$ is the channel gain, $s(t)$ is the transmitted signal and $w(t)$ is the noise signal at the receiver. The hypothesis $H_{0}$ and $H_{1}$ mean the absence and presence of the target signal, respectively.

\section{A. The $K$ and $K_{G}$ Channel Models}

The fading amplitude $X$ undergoes multipath fading as a Rayleigh distribution or a Nakagami distribution, and shadowing as a gamma distribution. Therefore, the average power of $X$, which represents the shadowing effect, follows the gamma distribution. The PDF of $X$, denoted as $f_{X}(x)$, follows a $K$ distribution when the multipath fading is Rayleigh distributed, or a $K_{G}$ distribution when the multipath fading is Nakagami distributed.

For the $K$ distribution, $f_{X}(x)$ can be written as [17]:

$$
f_{X}(x)=\frac{4}{\Gamma(k) \sqrt{\Omega}}\left(\frac{x}{\sqrt{\Omega}}\right)^{k} K_{k-1}\left(\frac{2}{\sqrt{\Omega}} x\right), \quad x \geq 0
$$

where $K_{v}(\cdot)$ is the $v$ th-order modified Bessel function of the second kind, $k$ is the shaping parameter, $\Omega$ represents the scale parameter which is also the mean signal power given as $\Omega=$ $\mathbb{E}\left[X^{2}\right] / k,{ }^{1}$ and $\Gamma(\cdot)$ is the standard gamma function. For the $K_{G}$ distribution, $f_{X}(x)$ can be written as [16]:

$$
f_{X}(x)=\frac{4 m^{\frac{\beta+1}{2}} x^{\beta}}{\Gamma(m) \Gamma(k) \Omega^{\frac{\beta+1}{2}}} K_{\alpha}\left[2\left(\frac{m}{\Omega}\right)^{\frac{1}{2}} x\right], \quad x \geq 0
$$

where $m$ is Nakagami fading parameter, $\alpha=k-m$, and $\beta=k+m-1$. The $K_{G}$ distribution reduces to a $K$ distribution when $m=1$. Moreover, as $m \rightarrow \infty$ and $k \rightarrow \infty$, the $K_{G}$ model tends to a non-fading case, i.e., the additive white Gaussian noise (AWGN) channel.

\section{B. Energy Detector Basics}

The energy detector measures the energy of the received signal $r(t)$ within a pre-defined bandwidth $W$ over time period $T$. It is important to note that the time-bandwidth product is given by $u=T W$, which is an integer. After sampling and frequency domain operations, the collected energy $\Lambda$ (which is the test statistic of the energy detector) follows a central chi-square distribution with $2 u$ degrees of freedom under hypothesis $H_{0}$, or a non-central chi-square distribution with $2 u$ degrees of freedom under hypothesis $H_{1}$. The detector compares the test statistic $\Lambda$ with a pre-defined threshold $\lambda$. The false alarm probability $\left(P_{f}\right)$ and detection probability $\left(P_{d}\right)$ can be evaluated by $\operatorname{Pr}\left(\Lambda>\lambda \mid H_{0}\right)$ and $\operatorname{Pr}\left(\Lambda>\lambda \mid H_{1}\right)$, respectively, as follows [5]:

$$
\begin{gathered}
P_{f}=\frac{\Gamma\left(u, \frac{\lambda}{2}\right)}{\Gamma(u)}, \\
P_{d}=Q_{u}(\sqrt{2 \gamma}, \sqrt{\lambda})
\end{gathered}
$$

where $Q_{u}(\cdot, \cdot)$ is the generalized Marcum-Q function, $\Gamma(\cdot, \cdot)$ is the upper incomplete gamma function defined as $\Gamma(a, x)=$ $\int_{x}^{\infty} \alpha^{a-1} e^{-\alpha} d \alpha$ and $\Gamma(a, 0)=\Gamma(a)$, and $\gamma$ is the received SNR of the target signal.

To demonstrate the detection performance, the receiver operating characteristic (ROC) curves are typically used, which are plots of the detection probability versus the false alarm probability. When the threshold $\lambda$ varies from 0 to $\infty$, the ROC curve moves from the upper right point $(1,1)$ to the origin $(0,0)$. In other words, to draw the entire ROC curve, the range of $\lambda$ should vary from 0 to $\infty$. In a real communication system, the value of $\lambda$ should be determined by the system requirement. In the wireless literature (such as in references [18]-[21]), most research efforts select the threshold $\lambda$ such that the false alarm probability is bounded by a target value. As shown in (1), the false alarm probability depends on two parameters: time-bandwidth product $u$ and the threshold $\lambda$. Therefore, the selection of $\lambda$ is not related to SNR $\gamma$. If

\footnotetext{
${ }^{1} \mathbb{E}[\cdot]$ denotes the expectation.
} 
we examine the typical parameter values considered in the literature, the false alarm probability is set as a value between $10^{-1}$ to $10^{-2}$ [19]-[21], and also IEEE 802.22 standard recommends $P_{f}<0.1$ in the spectrum sensing [22]. The timebandwidth product $(u=T W)$ is chosen within the range from 1 to 25 [3], [5], [6]. As an example, we can achieve $P_{f} \leq 10^{-2}$ with $u=25$ at $\lambda \geq 78$.

When $\lambda$ varies from 0 to $\infty$, the false alarm probability $P_{f}$ can be easily calculated by using (1) for given $u$. On the other hand, the detection probability is determined by the value of $\lambda$ and the channel fading/shadowing. Therefore, in a fading/shadowing channel, it is essential to evaluate the average of $P_{d}$ over the distribution of $\gamma$. The subsequent two sections are devoted to the calculation of this probability for the no-diversity case and diversity cases, respectively.

\section{Average Detection Probability with NO-DIVERSITY RECEPTION}

The SNR per received symbol is $\gamma=X^{2} E_{s} / N_{0}$, where $E_{s}$ and $N_{0}$ are the transmission energy per symbol and the singlesided power spectral density of the AWGN, respectively. The average detection probability of the energy detector, $\overline{P_{d}}$, can be evaluated by averaging $P_{d}$ in (2) over the SNR range. This can be expressed mathematically as follows:

$$
\begin{aligned}
\overline{P_{d}} & =\int_{0}^{\infty} Q_{u}(\sqrt{2 \gamma}, \sqrt{\lambda}) f_{\gamma}(\gamma) d \gamma \\
& =\int_{0}^{\infty} \int_{0}^{\infty} Q_{u}(\sqrt{2 \gamma}, \sqrt{\lambda}) f_{\gamma \mid Y=y}(\gamma) d \gamma f_{Y}(y) d y \\
& =\int_{0}^{\infty} P_{d}^{F a d}(y) f_{Y}(y) d y,
\end{aligned}
$$

where $Y$ is the SNR with only shadowing effect (i.e., multipath fading is excluded), which follows a gamma distribution [10]

$$
f_{Y}(y)=\frac{y^{k-1} e^{-\frac{y}{\Omega}}}{\Gamma(k) \Omega^{k}}, \quad y \geq 0
$$

and $P_{d}^{F a d}(y)=\int_{0}^{\infty} Q_{u}(\sqrt{2 \gamma}, \sqrt{\lambda}) f_{\gamma \mid Y=y}(\gamma) d \gamma$ is the average $^{2}$ detection probability with a specific $Y$ value. $P_{d}^{F a d}(y)$ for different applications under different multipath fading can be expressed using previous results in the literature [4] [5] [6], by replacing the $\bar{\gamma}$ 's in previous results with $y$.

Average detection probability $\overline{P_{d}}$ can be derived after averaging $P_{d}^{F a d}(y)$ by $f_{Y}(y)$ in (4). This approach appears mathematically more tractable than the direct integration of $P_{d}$ over $f_{\gamma}(\gamma)$ to find the overall average detection probability under the $K$ and $K_{G}$ channel models. Readers may refer to the Appendix for all derivations of the following results.

\section{A. Closed-form Average Detection Probability}

1) K Channel Model: The average detection probability over the $K$ channel model, $\overline{P_{d}^{K}}$, can be evaluated by replacing each $\bar{\gamma}$ of the average detection probability over Rayleigh distribution [6, eq. (9)] by $y$, and averaging over $f_{Y}(y)$ (see Appendix). Therefore, $\overline{P_{d}^{K}}$ can be expressed as in (6) which is

\footnotetext{
${ }^{2}$ Here the "average" means the average with respect to multipath fading.
}

on the next page where $U(; ;)$ is the confluent hypergeometric function of the second kind defined as [23, eq. (3.383.5)]:

$$
\int_{0}^{\infty} e^{-p x} x^{q-1}(1+a x)^{-v} d x=\frac{\Gamma(q)}{a^{q}} U\left(q ; q+1-v ; \frac{p}{a}\right)
$$

with $\operatorname{Re}\{q\}>0, \operatorname{Re}\{p\}>0, \operatorname{Re}\{a\}>0$ and $v$ a complex value. The result in expression (6) is valid for $u<k+1$.

2) $K_{G}$ Channel Model: The average detection probability over $K_{G}$ channel model, $\overline{P_{d}^{K_{G}}}$, can be evaluated by replacing each $\bar{\gamma}$ of the average detection probability over Nakagami- $m$ distribution [6, eq. (7)] by $y$, and averaging over $f_{Y}(y)$ (see Appendix). Therefore, $\overline{P_{d}^{K_{G}}}$ can be expressed as (7) on the next page, where $\left(\begin{array}{l}n \\ k\end{array}\right)$ is a binomial coefficient defined as $\left(\begin{array}{l}n \\ k\end{array}\right)=$ $\frac{n !}{k !(n-k) !},(x)_{s}$ is the Pochhammer symbol defined as $(x)_{s}=$ $\Gamma(x+s) / \Gamma(x)$, and $U(; ;)$ is given in (5). Expression (7) is valid for integer $m$. When $m=1$, this result is numerically equivalent to the one obtained in (6).

\section{B. Average Detection Probability Approximation}

One challenge in calculating the expressions (6) and (7) is the infinite sums. In the following, we use the expression (6) as an example. The expression (7) can be treated similarly.

The expression (6) can be rewritten as

$$
\overline{P_{d}^{K}}=e^{-\frac{\lambda}{2}} \sum_{n=0}^{u-2} \frac{1}{n !}\left(\frac{\lambda}{2}\right)^{n}+\sum_{n=0}^{\infty}(-1)^{n} a_{n}-\sum_{l=0}^{u-2} b_{l}
$$

where

$$
a_{n}=\frac{\left(\frac{\lambda}{2}\right)^{n} \Gamma(p) U\left(p ; k-n+1 ; \frac{1}{\Omega}\right)}{n ! \Gamma(k) \Omega^{k}}
$$

is the $n$th term of the auxiliary series $\sum_{n=0}^{\infty}(-1)^{n} a_{n}$, and

$$
b_{l}=\frac{e^{-\frac{\lambda}{2}}\left(\frac{\lambda}{2}\right)^{l} \Gamma(l+p) U\left(l+p ; k+1 ; \frac{1}{\Omega}\right)}{l ! \Gamma(k)(\Omega)^{k}}
$$

is the $l$ th term of the finite series $\sum_{l=0}^{u-2} b_{l}$, and $p=k-u+1$.

Theoretically, the auxiliary series converges as $n \rightarrow \infty$. When $\lambda$ is small, it is observed that the series converges for relatively small values of $n$, and thus, (6) can be accurately computed by using any mathematical software (e.g., MATHEMATICA [24]). When $\lambda$ is large, the sum requires the evaluation of $a_{n}$ for large $n$, and therefore, accurate computation of (6) is difficult (e.g., numerical underflow and overflow errors degrade the accuracy). For higher $\lambda$ values, the corresponding values of the average detection probability as well as the false alarm probability are very small (for instance, it can be less than $10^{-3}$ ). Thus, an approximation is needed for large $\lambda$.

Consequently, we define

$$
I_{K} \triangleq \int_{0}^{\infty}\left(\frac{1+y}{y}\right)^{u-1} e^{-\frac{\lambda}{2(1+y)}} f_{Y}(y) d y .
$$

Using $e^{-\frac{a}{b+x}}=\sum_{n=0}^{\infty} \frac{(-1)^{n} a^{n}}{n !(b+x)^{n}}$, we have

$$
\begin{aligned}
I_{K} & =\sum_{n=0}^{\infty} \frac{\left(-\frac{\lambda}{2}\right)^{n}}{n ! \Gamma(k) \Omega^{k}} \int_{0}^{\infty} y^{k-u}(1+y)^{-(n-u+1)} e^{-\frac{y}{2}} d y \\
& =\sum_{n=0}^{\infty}(-1)^{n} a_{n}
\end{aligned}
$$




$$
\begin{aligned}
\overline{P_{d}^{K}}=e^{-\frac{\lambda}{2}} \sum_{n=0}^{u-2} \frac{1}{n !}\left(\frac{\lambda}{2}\right)^{n}+\frac{1}{\Gamma(k) \Omega^{k}}[ & \sum_{n=0}^{\infty} \frac{(-1)^{n}\left(\frac{\lambda}{2}\right)^{n} \Gamma(k-u+1)}{n !} U\left(k-u+1 ; k-n+1 ; \frac{1}{\Omega}\right) \\
& \left.-e^{-\frac{\lambda}{2}} \sum_{n=0}^{u-2} \frac{\left(\frac{\lambda}{2}\right)^{n} \Gamma(n+k-u+1)}{n !} U\left(n+k-u+1 ; k+1 ; \frac{1}{\Omega}\right)\right] .
\end{aligned}
$$

$$
\begin{aligned}
\overline{P_{d}^{K_{G}}}=\frac{1}{\Gamma(k)}\left(\frac{m}{\Omega}\right)^{k}[ & e^{-\frac{\lambda}{2}} \sum_{s=0}^{\infty} \sum_{n=1}^{u-1} \frac{\left(\frac{\lambda}{2}\right)^{n+s}}{n ! s !} \frac{(m)_{s}}{(n+1)_{s}} \Gamma(s+k) U\left(s+k ; k-m+1 ; \frac{m}{\Omega}\right) \\
& +\sum_{t=0}^{\infty} \sum_{v=0}^{m-1} \frac{(-1)^{t}\left(\frac{\lambda}{2}\right)^{t+v} \Gamma(v+k)}{t ! v !}\left(\begin{array}{c}
m-1 \\
m-v-1
\end{array}\right) U\left(v+k ; k-t-m+2 ; \frac{m}{\Omega}\right) \\
& \left.+\sum_{s=0}^{\infty} \sum_{n=0}^{m-2} \sum_{a=0}^{n} \frac{(-1)^{s}\left(\frac{\lambda}{2}\right)^{a+s}}{a ! s !}\left(\begin{array}{c}
n \\
n-a
\end{array}\right) \Gamma(a+k+1) U\left(a+k+1 ; k-s-n+1 ; \frac{m}{\Omega}\right)\right] .
\end{aligned}
$$

where the second equality comes from (5). It can be seen that $I_{K}$ is the second term in (8). Note that the first and the third terms in (8) are finite sums and therefore, can be calculated exactly. Therefore, for accuracy of (8), it is essential to calculate $I_{K}$ accurately. In the following, convergence acceleration of (10) and an approximation method are given for $I_{K}$.

1) Convergence Acceleration: As $\lambda \rightarrow \infty$, a large number of terms in (10) need to be evaluated. This drawback can be avoided by using a convergence acceleration technique. The idea is to generate a new sequence by using $\left.a_{k}\right|_{k=0} ^{N}$. Consider the partial sums $s_{n}=\sum_{k=0}^{n}(-1)^{k} a_{k}$ for $n=0,1, \ldots, N-1$. The objective is to estimate the limit $s_{\infty}$ by using as few as possible partial sums. The $\epsilon$-algorithm [25] is a powerful convergence acceleration technique suitable for this purpose. The algorithm generates an array $\epsilon$ with $\epsilon_{-1}\left(s_{n}\right)=0$, $\epsilon_{0}\left(s_{n}\right)=s_{n}$, and

$$
\epsilon_{r+1}\left(s_{n}\right)=\epsilon_{r-1}\left(s_{n+1}\right)+\frac{1}{\epsilon_{r}\left(s_{n+1}\right)-\epsilon_{r}\left(s_{n}\right)}
$$

where $r(\geq 0)$ is an integer. The acceleration method starts with a partial sum of (10). It then estimates the converging point of $n$ through (11) while keeping adding adequate terms in (10) to reach the required accuracy.

2) Approximation: For large $\lambda$, we can approximate $I_{K}$ in (9) as

$$
I_{K} \approx \int_{0}^{\infty}\left(\frac{1+y}{y}\right)^{u-1} e^{-\frac{\lambda}{2 y}} f_{Y}(y) d y .
$$

After applying binomial expansion with some algebraic manipulations, (12) can be shown to be

$$
I_{K} \approx \frac{1}{\Gamma(k) \Omega^{k}} \sum_{s=0}^{u-1}\left(\begin{array}{c}
u-1 \\
s
\end{array}\right) \int_{0}^{\infty} y^{k-u+s} e^{-\left(\frac{\lambda}{2 y}+\frac{y}{\Omega}\right)} d y .
$$

With the aid of [23, eq. (3.478.4)], $I_{K}$ can be evaluated as

$$
I_{K} \approx \frac{2}{\Gamma(k) \Omega^{k}} \sum_{s=0}^{u-1}\left(\begin{array}{c}
u-1 \\
s
\end{array}\right)\left(\frac{\lambda \Omega}{2}\right)^{\frac{p}{2}} K_{p}\left(\sqrt{\frac{2 \lambda}{\Omega}}\right)
$$

where $p=k-u+s+1$. As comparing with (10), expression (13) consists of a finite summation with $K_{p}(\cdot)$, which is available in most mathematical software.
The approximation is given to the second term of (8). The first and the third terms of (8) are finite sums and therefore, can be calculated exactly. So, the accuracy of the average detection probability depends on the accuracy of the approximation. For instance, a four decimal points accuracy is achieved in the average detection probability when a four decimal points accuracy is achieved in $I_{K}$.

3) Error Analysis: The error term on $I_{K}$ due to the approximation given in (13) can be written as

$$
\mathcal{E}=\frac{1}{\Gamma(k) \Omega^{k}} \int_{0}^{\infty}\left(\frac{1+y}{y}\right)^{u-1} y^{k-1} e^{-\frac{y}{\Omega}}\left(e^{-\frac{\lambda}{2(1+y)}}-e^{-\frac{\lambda}{2 y}}\right) d y .
$$

It should be noted that the approximation is applied to evaluate the average detection probabilities at higher $\lambda$ values. When $\lambda$ goes to larger values, the behavior of $\mathcal{E}$ in (14) can be represented as

$$
\begin{gathered}
\mathcal{E} \rightarrow \frac{\left(\frac{2}{\lambda}\right)^{d}}{\Gamma(k) \Omega^{k}} \int_{0}^{\infty}\left(\frac{1+y}{y}\right)^{u-1} y^{k-1} e^{-\frac{y}{\Omega}}\left((1+y)^{d}-y^{d}\right) d y \\
=\frac{2^{d}}{\Gamma(k) \Omega^{k}}\left[\Gamma(p) U\left(p ; k+d+1 ; \frac{1}{\Omega}\right)\right. \\
\left.\quad-\Gamma(p+d) U\left(p+d ; k+d+1 ; \frac{1}{\Omega}\right)\right] \lambda^{-d}
\end{gathered}
$$

where $d=2$ and $p=k-u+1$. It can be seen that, for any $k, u$ and $\Omega$, the error exponentially decreases with high $\lambda$. Further, it is important to check the effect of error for higher average SNR values (i.e., $\Omega \rightarrow \infty$ ). When $x \rightarrow 0^{+}$, the confluent hypergeometric function of the second kind has asymptotic formula: $U(a ; c ; x) \approx \frac{\Gamma(c-1)}{\Gamma(a)} x^{1-c}, c>1$ [26]. For higher average SNR, it can be shown that $\mathcal{E} \rightarrow 0$, because the term in the square bracket of (15) goes to zero as $\Omega \rightarrow \infty$. Since the approximation (13) consists of finite sums, numerical values can be readily evaluated with mathematical software.

\section{Average Detection Probability with Diversity RECEPTION}

In this section, we discuss the performance of an energy detector with diversity combining methods. We consider $L$ 
diversity branches, which are independent and modeled with the $K$ distribution. First, we consider independent and identically distributed (i.i.d.) diversity branches, which have the same instantaneous shadowing in each branch. In this case, we can reapply the averaging technique (3). Next, we derive an accurate expression for the average detection probability under non-identical instantaneous shadowing. We mainly focus on two diversity techniques such as maximal-ratio combining (MRC) and selection combining (SC).

\section{A. Diversity branches with identical instantaneous shadowing}

1) Maximal Ratio Combining (MRC): The MRC receiver combines all the diversity branches weighted with their corresponding complex fading gains. The instantaneous SNR of the combiner output is thus $\gamma^{M R C}=\gamma_{1}+\gamma_{2}+\ldots+\gamma_{L}$. Since energy detector compares the received energy after the $L$ i.i.d. branches are combined, the expressions of the instantaneous false alarm and detection probabilities at the output of MRC for AWGN channels are the same as (1) and (2), respectively. To get the overall average detection probability, $P_{d}$ should then be averaged over the Rayleigh fading first, and then averaged over the shadowing. The PDF of $\gamma^{M R C}$ for i.i.d. Rayleigh fading channels is given by [10]

$$
f_{\gamma^{M R C}}(x)=\frac{x^{L-1} e^{-\frac{x}{\bar{\gamma}}}}{\Gamma(L) \bar{\gamma}^{L}}
$$

where $\bar{\gamma}$ is the average SNR in any branch (note that the "averaging" is on fading only, excluding shadowing). The $f_{\gamma^{M R C}}(x)$ in (16) is similar to the PDF of $\gamma$ under Nakagami fading in no-diversity. Therefore, after averaging on Rayleigh fading, the $P_{d}$ under MRC $\left(P_{d}^{M R C}\right)$ can be obtained from the average detection probability for a Nakagami channel with no diversity (i.e., by replacing $m$ by $L$ and $y$ by $L y$ in (18) in the Appendix). Therefore, the overall average detection probability for the $K$ channel model with MRC, $\overline{P_{d}^{M R C}}$, can be obtained by averaging the "average detection probability for a Nakagami channel with no diversity" over a gamma distribution, which is exactly the expression $\overline{P_{d}^{K_{G}}}$ in (7), but with $m$ replaced by $L$ and $\Omega$ replaced by $L \Omega$.

2) Selection Combining (SC): The combiner selects the branch having the strongest SNR among all diversity branches. The instantaneous SNR at the output of the combiner is $\gamma^{S C}=\max \left\{\gamma_{1}, \gamma_{2}, \ldots, \gamma_{L}\right\}$, where $\gamma_{k}$ is the SNR in the $k$ th branch. The PDF of $\gamma^{S C}$, without shadowing, is given in [5, eq. (29)]. Replacing each $\bar{\gamma}$ in [5, eq. (30)] by $y$ and averaging it over $f_{Y}(y)$, the average detection probability for $\mathrm{SC}$ diversity scheme under the $K$ channel model, $\overline{P_{d}^{S C}}$, can be evaluated as:

$$
\overline{P_{d}^{S C}}=L \sum_{p=0}^{L-1} \frac{(-1)^{p}}{p+1}\left(\begin{array}{c}
L-1 \\
p
\end{array}\right) \overline{P_{d}^{K}}\left(\frac{\Omega}{p+1}\right)
$$

where $\overline{P_{d}^{K}}\left(\frac{\Omega}{p+1}\right)$ is the $\overline{P_{d}^{K}}$ in (6) with the replacement of each $\Omega$ by $\left(\frac{\Omega}{p+1}\right)$.

Similarly, square-law combining (SLC) and square-law selection diversity techniques, which are frequently used in the cooperative spectrum sensing in cognitive radio networks, can be analyzed using the results in [6].

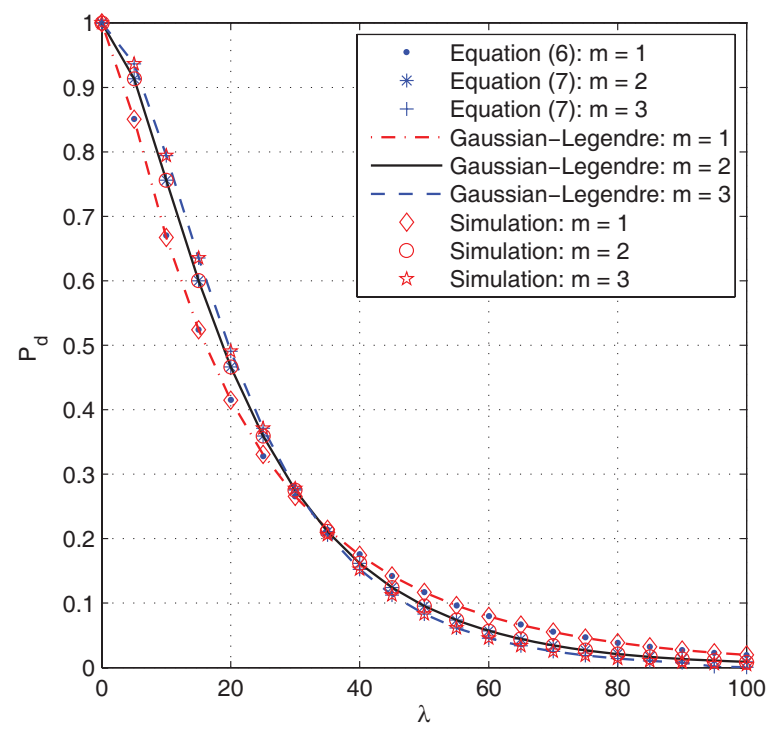

Fig. 1. Comparison of analytical expressions (6) and (7) with numerical approximations by the Gaussian-Legendre method, and Monte Carlo simulations $(u=2, k=5.5$ and $\bar{\gamma}=10 \mathrm{~dB})$.

\section{B. Diversity branches with non-identical instantaneous shad- owing}

When the diversity branches have non-identical instantaneous shadowing, PDFs of several diversity schemes have complicated mathematical expressions (e.g., MRC), or exact PDFs of some cases (e.g., EGC) are difficult to derive in closed-form [16]. The previous averaging technique (based on the assumption of identical instantaneous shadowing among all branches) cannot be applied in this case. In the following, we introduce an alternative method which can be applied with MRC.

The PDF of $\gamma^{M R C}$ under $K_{G}$ channel model is given in [16, eq. (11)]. Therefore, the PDF of $\gamma^{M R C}$ under $K$ channel model can be derived as (note that $m=1$ )

$f_{\gamma^{M R C}}(x)=\mathcal{F}(m=1, k, L) \Xi^{\frac{1+\tau}{2}} L^{\frac{1-\tau}{2}} x^{\frac{\tau-1}{2}} I_{\tau-1}(2 \sqrt{\Xi L x})$

where $\mathcal{F}(m=1, k, L)$ and $\tau$ are defined in [16, eq. (10)], and $\Xi=\frac{k}{\bar{\gamma}}$. The average detection probability can be written as

$$
\begin{aligned}
\overline{P_{d}^{M R C}=}= & \mathcal{F}(1, k, L) \Xi^{\frac{1+\tau}{2}} L^{\frac{1-\tau}{2}} \\
& \int_{0}^{\infty} x^{\tau} I_{\tau-1}(2 \sqrt{\Xi L} x) Q_{u}(\sqrt{2} x, \sqrt{\lambda}) d x .
\end{aligned}
$$

To the best of our knowledge, the integral in (17) cannot be solved in closed form in general. In some particular scenarios, such as $u=1, L=2$, and $k=1$ (i.e., one sample per real or quadrature component, dual-branch MRC, and severe fading and shadowing), $\overline{P_{d}^{M R C}}$ may be solved in closed-form with the aid of [27, eq. (5)], [28, eq. (2.15.20.7)] and [27, eq. (54)]. In Appendix C, an approximation is given for (17) by applying Gaussian integration methods as a generalized result. Similarly, the average detection probability over SLC can also be derived. This formulation is valid for $K_{G}$ channel model as well. 
TABLE I

NUMBER OF TERMS REQUIRED TO GET THE ACCURACY UP TO FOUR DECIMAL POINTS.

\begin{tabular}{|c|c|c|c|c|}
\cline { 2 - 5 } \multicolumn{1}{c|}{} & \multicolumn{4}{c|}{ Converging Point of $(n)$} \\
\cline { 2 - 5 } \multicolumn{1}{c|}{} & $\lambda=25$ & $\lambda=50$ & $\lambda=75$ & $\lambda=100$ \\
\hline Equation (6) & 20 & 50 & 84 & 114 \\
\hline$\epsilon-$ Algorithm & 11 & 15 & 19 & 23 \\
\hline Gaussian-Legendre & 38 & 38 & 38 & 38 \\
\hline
\end{tabular}

\section{Numerical and Simulation Results}

\section{A. Validation of the Analysis}

Analytical results in (6) and (7) are verified by numerical approximation $^{3}$ through the Gaussian-Legendre method and Monte Carlo simulations, as shown in Fig. 1.

The expressions (6) and (7) are calculated by MATHEMATICA software package [24], as shown in Fig. 1 by legend "Equation (6)" and "Equation (7)". Since the number of terms to sum up to achieve the required accuracy (e.g., up to four decimal points) increases with the increase of $\lambda$, we use an acceleration method, in which an $\epsilon$-algorithm [25] is applied to calculate the alternative series in (6) and (7). As an example, the converging points of $n$ (which reaches the required accuracy) for different $\lambda$ values are given in Table I, for the equation (6), the $\epsilon$-algorithm, and the GaussianLegendre method used in the numerical approximations.

Numerical integration is performed with the GaussianLegendre (Gaussian quadrature) method, which is explained here briefly. If an integral is in the form $\int_{-1}^{1} f(x) d x$, the Gaussian quadrature rule can be applied as

$$
\int_{-1}^{1} f(x) d x \approx \sum_{i=1}^{n} w_{i} f\left(x_{i}\right)
$$

by a suitable choice of the nodes (Legendre points) $x_{i}$ 's and weights $w_{i}$ 's $(i=1, \ldots, n)$, where $n$ is the number of nodes. The integral (9) in the form of $\int_{0}^{\infty} h(y) d y$ can be transformed to the form $\int_{0}^{\frac{\pi}{2}} \phi(\theta) d \theta$ by using the substitution $y=\tan \theta$. Further, it can be transformed to the form $\int_{-1}^{1} f(x) d x$ using the substitution $\theta=\frac{\pi}{4} x+\frac{\pi}{4}$. With the help of the three-term recurrence relation, nodes $x_{i}$ and weights $w_{i}$ can be computed from the associated eigenvalues and eigenvectors [29, Table 25.4]. Therefore, the Gaussian-Legendre method is suitable for $I_{K}$ to evaluate (6) numerically. A similar method can be applied to evaluate (7).

Fig. 1 shows that the analytical results in (6) and (7) match well with the simulations, and the Gaussian-Legendre method provides an accurate approximation.

\section{B. Accuracy of the Approximation for $I_{K}$}

In Fig. 2, we plot $I_{K}$ vs $\lambda$ for the $I_{K}$ definition (9) and the approximation in (13). The approximation acts as a lower bound for all $\lambda$ 's and the bound gets tighter when $\lambda$ increases from 0 to $\infty$ (i.e., for large $\lambda$ 's). As an example, we can get accuracy of four decimal points when $\lambda>150$ with $u=3, k=5.5$ and $\Omega=1$. However, it should be noted that for small $\lambda$ 's (practical threshold range), (6) and

\footnotetext{
${ }^{3}$ Note that the numerical approximation is only for numerical calculation of the expression (6) and (7). On the other hand, the expression (13) shows closed-form approximation for (6).
}

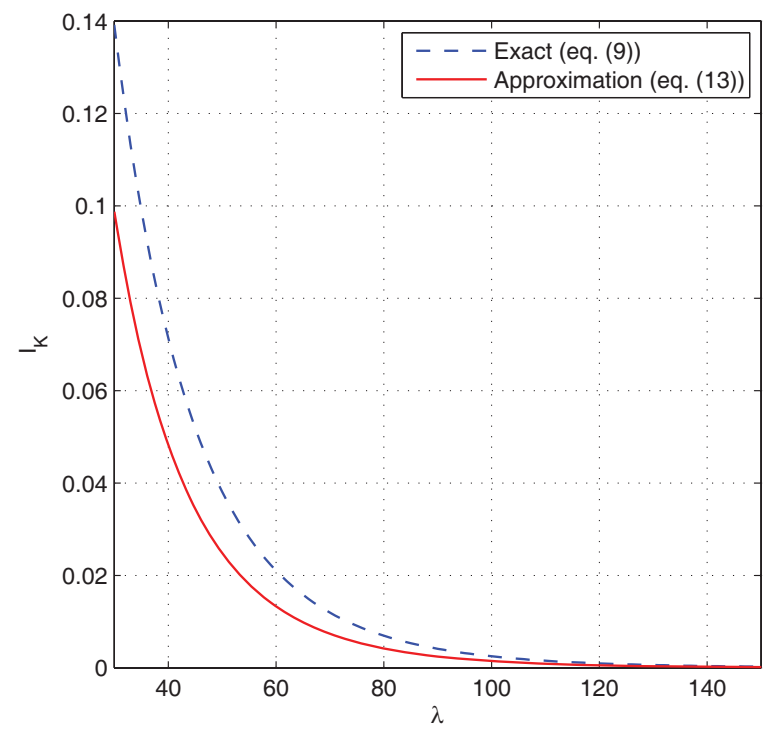

Fig. 2. Comparison of exact $I_{K}$ in (9) with Approximation in (13).

(7) can be used directly to evaluate the accurate detection probabilities without approximation. The approximation helps for asymptotic analysis.

\section{Impact of the Shadowing and Fading}

Fig. 3 shows the ROC curves (which are illustrated by $P_{d}$ vs $P_{f}$ ) for the $K$ channel model with different $k$ and average SNR $\bar{\gamma}$ with $u=1$. With the increase of $k$, an improvement in the detection probability is observed. This is because increasing $k$ diminishes the shadowing effect. When $k \rightarrow \infty$, the channel is a Rayleigh fading model.

For $K_{G}$ channel, Fig. 4 shows effect of multipath fading with fixed shadowing $(k=5.5)$. Different $m$ values are taken, with $\bar{\gamma}=5,10 \mathrm{~dB}$ and $u=1$. For larger $m$, the receiver has a higher detection probability with a lower false alarm probability, i.e., the channel fading conditions improve with the reduced fluctuations of the signal strength.

\section{Performance in Diversity Receptions}

The performance of MRC and SC diversity schemes with different number $L$ of diversity branches, which have the same instantaneous shadowing, is illustrated in Fig. 5. There is an obvious diversity gain in the case of diversity systems compared to no-diversity system (the case with $L=1$ ). Further, MRC always outperforms SC.

\section{E. Shadowing Effect}

Further, it is important to notice the differences between Rayleigh-lognormal distribution and $K$ distribution. In general, the performance of shadowing effect is compared with respect to shadowing standard deviation $\sigma_{\Omega}$ and mean $\mu_{\Omega}$ in lognormal distribution [10, eq. (2.200)]. The relationships between the parameters in Rayleigh-lognormal distribution $\left(\sigma_{\Omega}\right.$ and $\left.\mu_{\Omega}\right)$ and parameters in $K$ distribution $(k$ and $\Omega$ ) can be obtained in [11].

Fig. 6 shows the ROC curves of the energy detector for $\sigma_{\Omega} \approx 4.5 \mathrm{~dB}, 8 \mathrm{~dB}$ and $13 \mathrm{~dB}$ to represent urban area, 


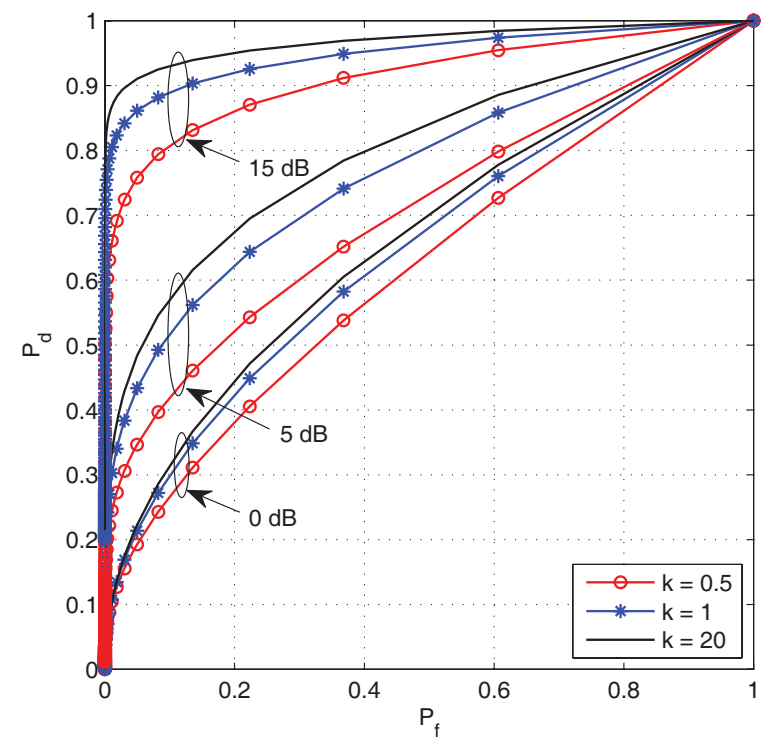

Fig. 3. ROC curves for the $K$ channel model with different $k(u=1$, $\bar{\gamma}=0,5,15 \mathrm{~dB})$.



Fig. 4. ROC curves for $K_{G}$ channel model with different fading parameters, $m(k=5.5, u=1, \bar{\gamma}=5,10 \mathrm{~dB})$.

typical microcell and worst case of macrocell, respectively. The respective $k$ and $\mu_{\Omega}$ values for $\sigma_{\Omega} \approx 4.5,8.0,13 \mathrm{~dB}$ are $k \approx 1.35,0.63,0.35$ and $\mu_{\Omega} \approx 2.5155,-3.2254,-9.893 \mathrm{dBm}$ with $\Omega=2$, respectively. Fig. 6 shows that the $K$ distribution is a well-approximated model for the the Rayleigh-lognormal distribution. Similar observations can also be made for the $K_{G}$ distribution and the Nakagami-lognormal distribution. These results are omitted here due to the space limit.

\section{CONCLUSION}

We study the performance of an energy detector under both multipath fading and shadowing effects, effects that are well modeled by the $K$ and $K_{G}$ distributions. Exact representations of the average detection probabilities are derived, along with the approximations for large $\lambda$ values. As well, convergence

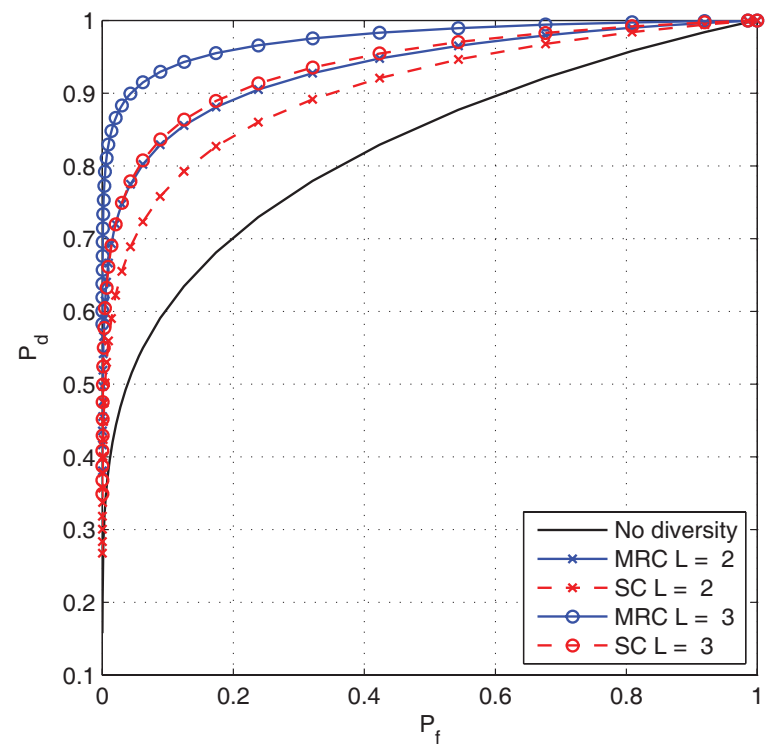

Fig. 5. ROC curves for $L$-branch MRC and SC diversity receptions with $K$ channel model $(u=3, k=6, \bar{\gamma}=5 \mathrm{~dB})$.

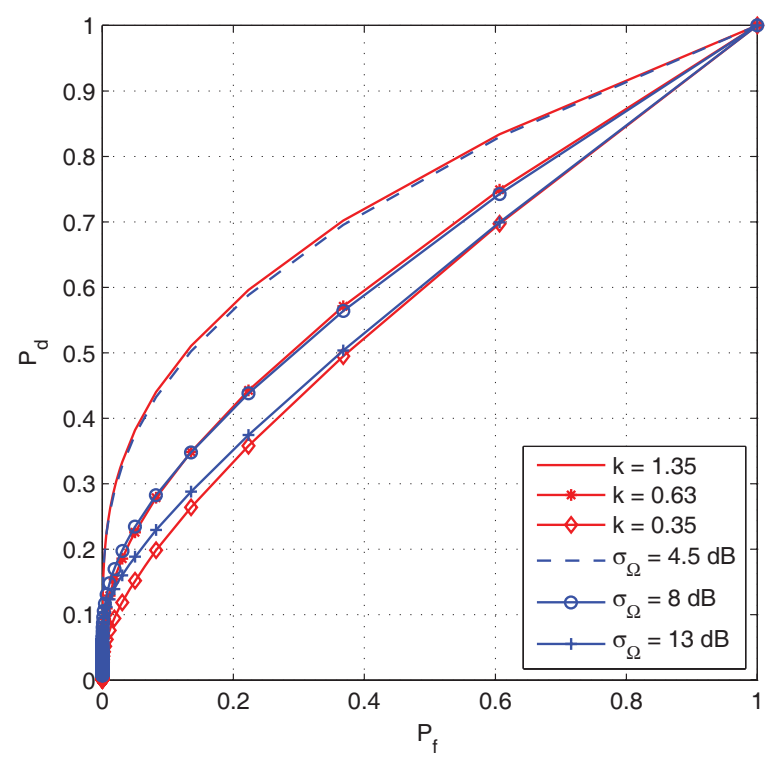

Fig. 6. Comparison of $K$ channel model with Rayleigh-lognormal channel.

acceleration based on the $\epsilon$-algorithm is suggested to efficiently compute the infinite series representation. We also find that the numerically efficient Gaussian-Legendre quadrature is useful for numerical approximations. Our results show that the detection probability increases with the fading parameter $m$ and shadowing parameter $k$. The reason is that as $m \rightarrow \infty$ and $k \rightarrow \infty$, the channel approaches the AWGN channel. Furthermore, when diversity receptions such as MRC and SC are used to boost the performance of the energy detector, we derive their performance under identically and non-identically shadowed diversity branches. The ROC reveals the effect of diversity advantage, and, as expected, MRC improves the performance of the energy detector more than SC. Finally, numerical results for the Rayleigh-lognormal distribution and the $K$ distribution are compared to confirm that the latter models the shadowing effect well. Our results provide comprehensive 
performance evaluation of the energy detector with or without diversity reception in wireless channels subject to fading and shadowing.

\section{APPENDIX}

\section{A. Derivation of $\overline{P_{d}^{K}}$}

$\overline{P_{d}^{K}}$ can be evaluated as $\int_{0}^{\infty} P_{d}^{F a d}(y) f_{Y}(y) d y$ where $f_{Y}(y)$ is in (4). $P_{d}^{F a d}(y)$ can be evaluated by setting $\sigma^{2}=1, a=2$, $N=2 u$, and by replacing each $\bar{\gamma}$ by $y$ in the average detection probability over Rayleigh distribution in [6, eq. (9)] , which yields

$$
\begin{aligned}
P_{d}^{F a d}(y)= & e^{-\frac{\lambda}{2}} \sum_{n=0}^{u-2} \frac{1}{n !}\left(\frac{\lambda}{2}\right)^{n}+\left(\frac{1+y}{y}\right)^{u-1} \\
& \cdot\left[e^{-\frac{\lambda}{2(1+y)}}-e^{-\frac{\lambda}{2}} \sum_{n=0}^{u-2} \frac{1}{n !}\left(\frac{\lambda y}{2(1+y)}\right)^{n}\right] .
\end{aligned}
$$

After applying series summation for exponential function, and with some algebraic manipulations, $\overline{P_{d}^{K}}$ can be written as

$$
\begin{aligned}
\overline{P_{d}^{K}} & =e^{-\frac{\lambda}{2}} \sum_{n=0}^{u-2} \frac{1}{n !}\left(\frac{\lambda}{2}\right)^{n} \\
& +\frac{1}{\Gamma(k) \Omega^{k}} \sum_{n=0}^{\infty} \frac{\left(-\frac{\lambda}{2}\right)^{n}}{n !} \int_{0}^{\infty} y^{k-u}(1+y)^{u-n-1} e^{-\frac{y}{\Omega}} d y \\
& -\frac{e^{-\frac{\lambda}{2}}}{\Gamma(k) \Omega^{k}} \sum_{n=0}^{u-2} \frac{\left(\frac{\lambda}{2}\right)^{n}}{n !} \int_{0}^{\infty} y^{n+k-u}(1+y)^{u-n-1} e^{-\frac{y}{\Omega}} d y .
\end{aligned}
$$

$\overline{P_{d}^{K}}$ can be derived as (6) with the aid of (5).

\section{B. Derivation of $\overline{P_{d}^{K_{G}}}$}

$\overline{P_{d}^{K_{G}}}$ can be evaluated as $\int_{0}^{\infty} P_{d}^{F a d}(y) f_{Y}(y) d y$ where $P_{d}^{F a d}(y)$ can be written using [6, eq. (7)]. We use series summation of ${ }_{1} F_{1}(a ; c ; x)=\sum_{n=0}^{\infty} \frac{(a)_{n} x^{n}}{(c)_{n} n !}$ [26, eq. (10.1)] and $L_{n}(x)=\sum_{k=0}^{n}(-1)^{k}\left(\begin{array}{c}n \\ n-k\end{array}\right) \frac{x^{k}}{k !}$ [23, eq. (8.970.1)], where ${ }_{1} F_{1}(\cdot ; \cdot ; \cdot)$ and $L_{n}(\cdot)$ are the confluent hypergeometric function and the Laguerre polynomial of degree $n$, respectively. After some algebraic manipulations, equivalent $P_{d}^{F a d}(y)$ can be expressed as ${ }^{4}$

$$
\begin{aligned}
P_{d}^{F a d}(y) & =\sum_{v=0}^{m-1} \frac{\left(\begin{array}{c}
m-1 \\
m-v-1
\end{array}\right)\left(\frac{\lambda}{2}\right)^{v}}{v ! m^{1-m}} e^{-\frac{\lambda}{2} \frac{m}{m+y}} y^{v}(m+y)^{1-m-v} \\
+ & \sum_{n=0}^{m-2} \sum_{a=0}^{n} \frac{\left(\begin{array}{c}
n \\
n-a
\end{array}\right)\left(\frac{\lambda}{2}\right)^{a}}{a ! m^{-n}} e^{-\frac{\lambda}{2} \frac{m}{m+y}} y^{a+1}(m+y)^{-n-a-1} \\
+ & \sum_{s=0}^{\infty} \sum_{n=1}^{u-1} \frac{e^{-\frac{\lambda}{2}}\left(\frac{\lambda}{2}\right)^{n+s} m^{m}}{n ! s !} \frac{(m)_{s}}{(n+1)_{s}} y^{s}(m+y)^{-s-m}
\end{aligned}
$$

Applying series summation for exponential function and averaging it over $f_{Y}(y)$, (18) can be re-written as (19) on the top of the next page. $\overline{P_{d}^{K_{G}}}$ can be evaluated as (7) with the aid of (5).

\footnotetext{
${ }^{4}$ The expression in $[6$, eq. (8)] has a typo. The power of the exponential term should be $-\frac{\lambda \beta}{2 \sigma^{2}}$.
}

C. Derivation of $\overline{P_{d}^{M R C}}$ with Different Instantaneous Shadowing

With the definition of the generalized Marcum- $Q$ function [30, eq. (1)] and substituting $y=(\gamma-\sqrt{\lambda}), Q_{u}(\sqrt{2 \gamma}, \sqrt{\lambda})$ in (17) can be written as

$$
\begin{aligned}
Q_{u}(\sqrt{2 \gamma}, \sqrt{\lambda})= & \frac{1}{(2 \gamma)^{\frac{u-1}{2}}} \int_{0}^{\infty}(y+\sqrt{\lambda})^{u} e^{-\frac{(y+\sqrt{\lambda})^{2}+2 \gamma}{2}} \\
& \cdot I_{u-1}(\sqrt{2 \gamma}(y+\sqrt{\lambda})) d y .
\end{aligned}
$$

After applying the Gaussian-Laguerre integration, (20) can be accurately approximated as

$$
Q_{u}(\sqrt{2 \gamma}, \sqrt{\lambda}) \approx \frac{e^{-\gamma}}{\gamma^{\frac{u-1}{2}}} \sum_{i=1}^{N} \Delta_{i} I_{u-1}\left(\sqrt{2 \gamma}\left(t_{i}+\sqrt{\lambda}\right)\right)
$$

where $\Delta_{i}=\frac{w_{i} e^{t_{i}}\left(t_{i}+\sqrt{\lambda}\right)^{u} e^{-\frac{\left(t_{i}+\sqrt{\lambda}\right)^{2}}{2}}}{2^{\frac{u-1}{2}}}$, and $w_{i}$ and $t_{i}$ are weight factors and abscissas $(i=1, \ldots, N)$ of the Gaussian Laguerre integration, respectively [29, Table 25.9]. Therefore, the average detection probability under MRC given in (17) can be written as

$$
\begin{aligned}
\overline{P_{d}^{M R C}} & \approx 2 \mathcal{F}(1, k, L) \Xi^{\frac{1+\tau}{2}} L^{\frac{1-\tau}{2}} \sum_{i=1}^{N} \Delta_{i} \int_{0}^{\infty} y^{\tau-u+1} \\
& \cdot e^{-y^{2}} I_{u-1}\left(\sqrt{2}\left(t_{i}+\sqrt{\lambda}\right) y\right) I_{\tau-1}(2 \sqrt{\Xi L} y) d y .
\end{aligned}
$$

With the aid of [28, eq. (2.15.20.7)], $\overline{P_{d}^{M R C}}$ can be derived as

$$
\begin{aligned}
\overline{P_{d}^{M R C} \approx 2} & \mathcal{F}(1, k, L) \Xi^{\frac{1+\tau}{2}} L^{\frac{1-\tau}{2}} \\
& \cdot \sum_{i=1}^{N} \frac{\Delta_{i}\left(t_{i}+\sqrt{\lambda}\right)^{u-1}(\Xi L)^{\frac{\tau-1}{2}}}{2^{\frac{u+1}{2}} \Gamma(\tau)} \\
& \cdot \sum_{j=0}^{\infty} \frac{\Gamma(\tau+j)}{j ! \Gamma(u+j)}\left(\frac{t_{i}+\sqrt{\lambda}}{\sqrt{2}}\right)^{2 j} \\
& \cdot{ }_{2} F_{1}\left(-j, 1-u-j ; \tau ; \frac{2 \Xi L}{\left(t_{i}+\sqrt{\lambda}\right)^{2}}\right) .
\end{aligned}
$$

Further, when $u=\tau, \overline{P_{d}^{M R C}}$ can be derived in simple form as $[28$, eq. $(2.15 .20 .8)]$

$$
\begin{array}{r}
\overline{P_{d}^{M R C}} \approx \mathcal{F}(1, k, L) \Xi^{\frac{1+\tau}{2}} L^{\frac{1-\tau}{2}} \sum_{i=1}^{N} \Delta_{i} e^{\frac{\left(t_{i}+\sqrt{\lambda}\right)^{2}+2 \Xi L}{2}} \\
\cdot I_{u-1}\left(\sqrt{2 \Xi L}\left(t_{i}+\sqrt{\lambda}\right)\right) .
\end{array}
$$

\section{REFERENCES}

[1] V. K. Bhargava and E. Hossain (editors), Cognitive Wireless Communication Networks. New York: Springer-Verlag, 2007.

[2] A. Rabbachin, T. Q. S. Quek, P. C. Pinto, I. Oppermann, and M. Z. Win, "UWB energy detection in the presence of multiple narrow-band interferers," in Proc. IEEE Int. Conf. Ultra-Wideband (ICUWB), 2007, pp. 857-862.

[3] H. Urkowitz, "Energy detection of unknown deterministic signals," Proc. IEEE, vol. 55, no. 4, pp. 523-531, Apr. 1967.

[4] V. I. Kostylev, "Energy detection of a signal with random amplitude," in Proc. IEEE Int. Conf. Commun. (ICC), 2002, pp. 1606-1610.

[5] F. F. Digham, M. S. Alouini, and M. K. Simon, "On the energy detection of unknown signals over fading channels," in Proc. IEEE Int. Conf. Commun. (ICC), 2003, pp. 3575-3579. 


$$
\begin{aligned}
\overline{P_{d}^{K_{G}}}=\frac{1}{\Gamma(k)(\Omega)^{k}}[ & \sum_{t=0}^{\infty} \sum_{v=0}^{m-1} \frac{(-1)^{t}\left(\frac{\lambda}{2}\right)^{t+v}\left(\begin{array}{c}
m-1 \\
m-v-1
\end{array}\right)}{t ! v ! m^{v}} \int_{0}^{\infty} y^{v+k-1}\left(1+\frac{y}{m}\right)^{-t-m-v+1} e^{-\frac{y}{\Omega}} d y \\
& +\sum_{s=0}^{\infty} \sum_{n=0}^{m-2} \sum_{a=0}^{n} \frac{(-1)^{s}\left(\frac{\lambda}{2}\right)^{a+s}\left(\begin{array}{c}
n \\
n-a
\end{array}\right)}{a ! s ! m^{a+1}} \int_{0}^{\infty} y^{a+k}\left(1+\frac{y}{m}\right)^{-s-n-a-1} e^{-\frac{y}{\Omega}} d y \\
& \left.+e^{-\frac{\lambda}{2}} \sum_{s=0}^{\infty} \sum_{n=1}^{u-1} \frac{\left(\frac{\lambda}{2}\right)^{n+s}}{n ! s ! m^{s}} \frac{(m)_{s}}{(n+1)_{s}} \int_{0}^{\infty} y^{s+k-1}\left(1+\frac{y}{m}\right)^{-m-s} e^{-\frac{y}{\Omega}} d y\right]
\end{aligned}
$$

[6] F. F. Digham, M. S. Alouini, and M. K. Simon, "On the energy detection of unknown signals over fading channels," IEEE Trans. Commun., vol. 55, no. 1, pp. 21-24, Jan. 2007.

[7] S. P. Herath and N. Rajatheva, "Analysis of equal gain combining in energy detection for cognitive radio over Nakagami channels," in Proc. IEEE Global Telecommun. Conf. (GLOBECOM), 2008.

[8] S. Atapattu, C. Tellambura, and H. Jiang, "Relay based cooperative spectrum sensing in cognitive radio networks," in Proc. IEEE Global Telecommn. Conf. (GLOBECOM), 2009.

[9] S. Atapattu, C. Tellambura, and H. Jiang, "Energy detection of primary signals over $\eta-\mu$ fading channels," in Proc. Int. Conf. Industrial \& Information Systems (ICIIS), Dec. 2009, pp. 118-122.

[10] G. L. Stüber, Principles of Mobile Communication, 2nd edition. Norwell, MA: Kluwer Academic Publishers, 2001.

[11] A. Abdi and M. Kaveh, " $K$ distribution: an appropriate substitute for Rayleigh-lognormal distribution in fading-shadowing wireless channels," Electron. Lett., vol. 34, no. 9, pp. 851-852, Apr. 1998.

[12] P. M. Shankar, "Error rates in generalized shadowed fading channels," Wireless Personal Commun., vol. 28, no. 3, pp. 233-238, Feb. 2004.

[13] A. Abdi and M. Kaveh, "Comparison of DPSK and MSK bit error rates for $K$ and Rayleigh-lognormal fading distributions," IEEE Commun. Lett., vol. 4, no. 4, pp. 122-124, Apr. 2000.

[14] P. M. Shankar, "Performance analysis of diversity combining algorithms in shadowed fading channels," Wireless Personal Commun., vol. 37, no. 1-2, pp. 61-72, Apr. 2006.

[15] P. S. Bithas, N. C. Sagias, P. T. Mathiopoulos, G. K. Karagiannidis, and A. A. Rontogiannis, "On the performance analysis of digital communications over generalized- $K$ fading channels," IEEE Commun. Lett., vol. 10, no. 5, pp. 353-355, May 2006.

[16] P. S. Bithas, P. T. Mathiopoulos, and S. A. Kotsopoulos, "Diversity reception over generalized- $K\left(K_{G}\right)$ fading channels," IEEE Trans. Wireless Commun., vol. 6, no. 12, pp. 4238-4243, Dec. 2007.

[17] P. Theofilakos, A. G. Kanatas, and G. P. Efthymoglou, "Performance of generalized selection combining receivers in $K$ fading channels," IEEE Commun. Lett., vol. 12, no. 11, pp. 816-818, Nov. 2008.

[18] B. H. Juang, G. Y. Li, and J. Ma, "Signal processing in cognitive radio," Proc. IEEE, vol. 97, no. 5, pp. 805-823, May 2009.

[19] G. Ganesan and Y. Li, "Cooperative spectrum sensing in cognitive radio, part I: two user networks," IEEE Trans. Wireless Commun. vol. 6, no. 6, pp. 2204-2213, June 2007.

[20] F. Penna, R. Garello, and M. Spirito, "Cooperative spectrum sensing based on the limiting eigenvalue ratio distribution in Wishart matrices," IEEE Commun. Lett., vol. 13, no. 7, pp. 507-509, July 2009.

[21] J. Lunden, V. Koivunen, A. Huttunen, and H. V. Poor, "Collaborative cyclostationary spectrum sensing for cognitive radio systems," IEEE Trans. Signal Process., vol. 57, no. 11, pp. 4182-4195, Nov. 2009.

[22] C. Stevenson, G. Chouinard, Z. Lei, W. Hu, S. Shellhammer, and W. Caldwell, "IEEE 802.22: the first cognitive radio wireless regional area network standard," IEEE Commun. Mag., vol. 47, no. 1, pp. 130 -138 , Jan. 2009.

[23] I. S. Gradshteyn and I. M. Ryzhik, Table of Integrals, Series, and Products, 6th edition. Academic Press, Inc., 2000.

[24] Wolfram, The Wolfram functions site. [Online]. Available: http://functions.wolfram.com, 2010.

[25] T. Sauter, "Computation of irregularly oscillating integrals," Appl. Numer. Math., vol. 35, no. 3, pp. 245-264, Nov. 2000.

[26] L. C. Andrews, Special Functions of Mathematics for Engineers, 2nd edition. New York: McGraw-Hill, 1992.

[27] A. H. Nuttall, "Some integrals involving the Q-function." Naval Underwater Systems Center (NUSC) technical report, Apr. 1972.

[28] A. P. Prudnikov, Y. A. Brychkov, and O. I. Marichev, Integrals and Series, vol. 2. Gordon and Breach Science Publishers, 1986.
[29] M. Abramowitz and I. A. Stegun (editors), Handbook of Mathematical Functions: With Formulas, Graphs, and Mathematical Tables. New York: Dover Publications, 1965.

[30] A. H. Nuttall, "Some integrals involving the $Q_{m}$-function." Naval Underwater Systems Center (NUSC) technical report, May 1974.

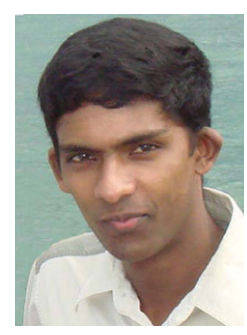

Saman Atapattu (S'06) received the B.Sc. degree in electrical and electronics engineering from the University of Peradeniya, Sri Lanka in 2003 and the M. Eng. degree in telecommunications from Asian Institute of Technology (AIT), Thailand in 2007. He is currently working towards the Ph.D. degree in electrical and computer engineering at the University of Alberta, Edmonton, AB, Canada.

His research interests include cooperative communications, cognitive radio networks, and performance analysis of communication systems.

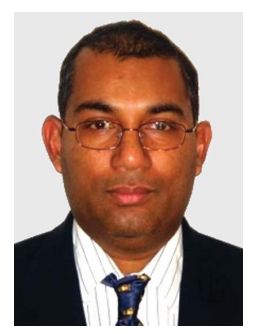

Chintha Tellambura (SM'02) received the B.Sc. degree (with first-class honor) from the University of Moratuwa, Sri Lanka, in 1986, the M.Sc. degree in Electronics from the University of London, U.K., in 1988, and the Ph.D. degree in Electrical Engineering from the University of Victoria, Canada, in 1993.

He was a Postdoctoral Research Fellow with the University of Victoria (1993-1994) and the University of Bradford (1995-1996). He was with Monash University, Australia, from 1997 to 2002. Presently, he is a Professor with the Department of Electrical and Computer Engineering, University of Alberta. His research interests include diversity and fading countermeasures, multiple-input multiple-output (MIMO) systems and space-time coding and orthogonal frequency division multiplexing (OFDM).

Prof. Tellambura is an Associate Editor for the IEEE TRANSACTIONS on COMmunications and the Area Editor for Wireless Communications Systems and Theory in the IEEE TRANSACTIONS ON WIRELESS COMMUNICATIONS. He was Chair of the Communication Theory Symposium in Globecom'05 held in St. Louis, MO.

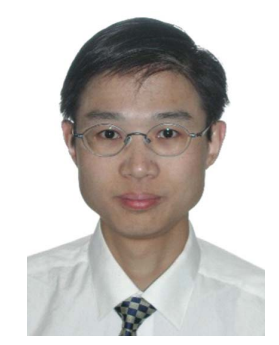

Hai Jiang (M'07) received the B.Sc. and M.Sc. degrees in electronics engineering from Peking University, Beijing, China, in 1995 and 1998, respectively, and the Ph.D. degree (with an Outstanding Achievement in Graduate Studies Award) in electrical engineering from the University of Waterloo, Waterloo, ON, Canada, in 2006.

Since July 2007, he has been an Assistant Professor with the Department of Electrical and Computer Engineering, University of Alberta, Edmonton, AB, Canada. His research interests include radio resource management, cognitive radio networking, and cross-layer design for wireless multimedia communications.

Dr. Jiang is an Associate Editor for the IEEE TRANSACTIONS ON VeHICUlaR TeChNOLOGY. He served as a Co-Chair for the General Symposium at the International Wireless Communications and Mobile Computing Conference (IWCMC) in 2007, the Communications and Networking Symposium at the Canadian Conference on Electrical and Computer Engineering (CCECE) in 2009, and the Wireless and Mobile Networking Symposium at the IEEE International Conference on Communications (ICC) in 2010. He received an Alberta Ingenuity New Faculty Award in 2008 and a Best Paper Award from the IEEE Global Communications Conference (GLOBECOM) in 2008. 